\section{Informações sobre mortalidade por causas externas e eventos de intenção indeterminada, Paraná, Brasil, 1979 a 2005}

\author{
Data on mortality from external causes and events \\ of undetermined intent, Paraná State, Brazil, \\ 1979 to 2005
}

11 a Regional de Saúde, Secretaria de Estado da Saúde do Paraná, Campo Mourão, Brasil.

2 Departamento de

Enfermagem, Universidade

Estadual de Maringá,

Maringá, Brasil.

${ }^{3}$ Departamento de Saúde

Coletiva, Universidade

Estadual de Londrina,

Londrina, Brasil.

${ }^{4}$ Instituto de Filosofia

e Ciências Humanas,

Universidade Estadual de

Campinas, Campinas, Brasil.

${ }_{5}$ Núcleo de Estudos de

População, Universidade

Estadual de Campinas,

Campinas, Brasil.

Correspondência

T. A. F. Mathias

Departamento de

Enfermagem, Universidade

Estadual de Maringá.

Av. Colombo 5790, Zona 7,

Maringá, $P R$

87020-900, Brasil.

tafmathias@uem.br

\section{Abstract}

This study analyzed the quality of data from the Mortality Information System (SIM) for deaths due to external causes in the State of Paraná, Brazil, 1979 to 2005, focusing on events of undetermined intent. Deaths were grouped in motor vehicle accidents, homicides, suicides, and events of undetermined intent, and proportional mortality and relative annual variation of rates over the three-year period were analyzed. Motor vehicle accidents (more than $30 \%$ of the total) were the most frequent causes of death throughout the period, and since 1997 homicides have become the second most frequent cause. Deaths due to events of undetermined intent caused by weapons (firearms or knives) decreased from 4.8\% in 1981 to $0.3 \%$ in 2005. Mortality rates for events of undetermined intent (overall) decreased from 14.9 deaths per 100,000 inhabitants in 1979-81 to 2.0 in 2003-05. Annual percentage variation was 13.1\% from 1980 to 1985, -6\% from 1996 to 2000, and $-11 \%$ from 2000 to 2004. The findings show the good quality of SIM data on external causes in the State of Paraná, allowing analyses with the potential to support programs to prevent injuries as well as health promotion measures.

External Causes; Homicide; Mortality; Information Systems
Elizabeth Mitiko Konno de Lozada 1

Thais Aidar de Freitas Mathias 2

Selma Maffei de Andrade 3

Tirza Aidar 4,5

As causas externas têm ganhado importância na mortalidade nas últimas décadas, principalmente devido à intensificação das mortes por homicídios entre 1980 e o final dos anos 90 , que respondiam, em 2004, por 39,1\% das mortes por causas externas no país 1 . Reflexo dos problemas urbanos contemporâneos, o crescimento das mortes violentas tem motivado estudos, com importância crescente no cenário nacional e internacional 2,3. Em geral esses estudos utilizam como fonte o banco de dados do Sistema de Informações sobre Mortalidade (SIM) do Ministério da Saúde, que tem como base a Declaração de Óbito (DO).

Quando o óbito decorre de causa externa, a DO é preenchida por perito legista que, muitas vezes, detalha somente a natureza da lesão (fratura, hemorragia, lacerações, entre outras), sem a identificação se acidente, homicídio ou suicídio, fundamental para a análise do perfil da mortalidade e orientação de ações preventivas nos diversos setores e níveis governamentais. Assim, é essencial conhecer o potencial e as limitações do SIM em relação às causas externas.

Para conhecer a qualidade do SIM em relação aos óbitos por causas externas no Estado do Paraná, entre 1979 e 2005, foi analisada a tendência temporal dos coeficientes de mortalidade proporcional por tipos de causas externas, focalizando os "eventos de intenção indeterminada". 


\section{Material e método}

Foram estudados os óbitos por causas externas de residentes no Paraná, extraídos do SIM (Departamento de Informática do SUS; http:// tabnet.datasus.gov.br/cgi/deftohtm.exe?sim/ cnv/extpr.def, acessado em 18/Jul/2007), agrupados em: acidentes de transporte, homicídios, suicídios, eventos de intenção indeterminada e outras causas externas. Óbitos classificados como intervenção legal foram previamente agregados aos homicídios conforme recomendação 3 . Entre os "eventos de intenção indeterminada" foram destacados: lesão por arma de fogo, por arma branca, outras lesões e lesões não especificadas (Figura 1).

Os óbitos foram agrupados em seis triênios: 1979/1981, 1984/1986, 1990/1992, 1995/1997, 1999/2001 e 2003/2005 com o objetivo de reduzir flutuações aleatórias. Os dados de população são provenientes dos censos de 1980, 1991, e 2000 e contagem de 1996, com estimativas do Instituto Brasileiro de Geografia e Estatística (IBGE). Os coeficientes de mortalidade significam a média dos óbitos por causas externas, em cada triênio, pela população no meio do período (por $100 \mathrm{mil}$ habitantes). Além da análise gráfica, a tendência da mortalidade foi analisada pela variação percentual anual (VA\%) dos coeficientes entre os triênios:

$$
V A \%=\frac{\frac{C_{t_{f}}-C_{t_{i}}}{C_{t_{i}}} \times 100}{t_{f}-t_{i}}
$$

Onde $C_{t}$ é o coeficiente de mortalidade do triênio $t ; t_{f}$ e $t_{i}$ são os anos centrais dos triênios final e inicial, respectivamente, ou seja, $t_{f}$ e $t_{i}$ assumem os valores 1980, 1985, 1991, 1996, 2000 ou 2004.

O projeto foi submetido à apreciação do Comitê Permanente de Ética em Pesquisa da Universidade Estadual de Maringá (parecer $n^{\circ}$. 350/2004).

\section{Resultados}

Os acidentes de transporte foram os principais provocadores de mortes por causas externas no Paraná, em todo o período, vindo em seguida, até 1996, os outros acidentes (como quedas, envenenamentos e afogamentos). A partir de 1997, observa-se aumento no percentual de óbitos por homicídios, que passam à segunda posição, atingindo, em 2005, 35,6\% (Figura 2).

A proporção de óbitos por eventos (todos) de intenção indeterminada diminuiu de 22,3\% em 1980 para $6,7 \%$ em 1985, chegando a $2,1 \%$ do total de óbitos por causas externas em 2005. A mortalidade proporcional por eventos de inten-

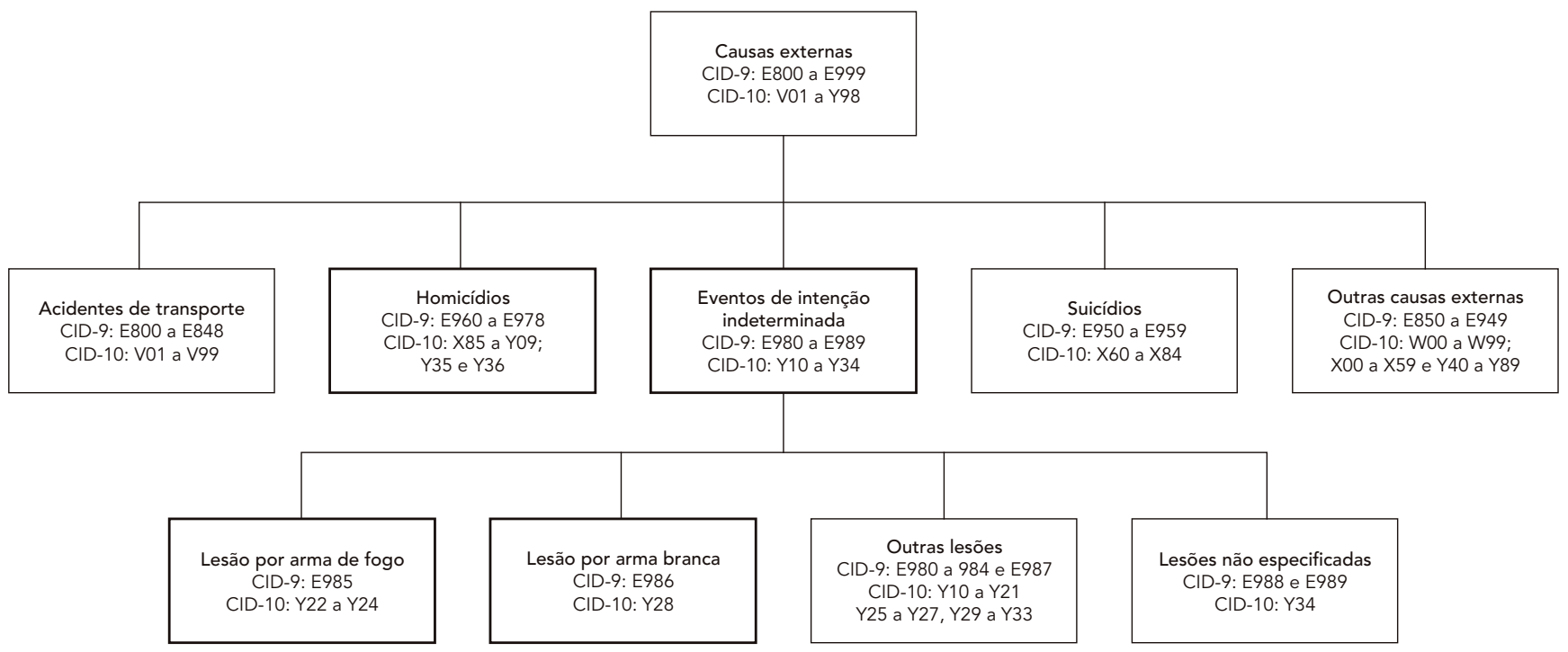




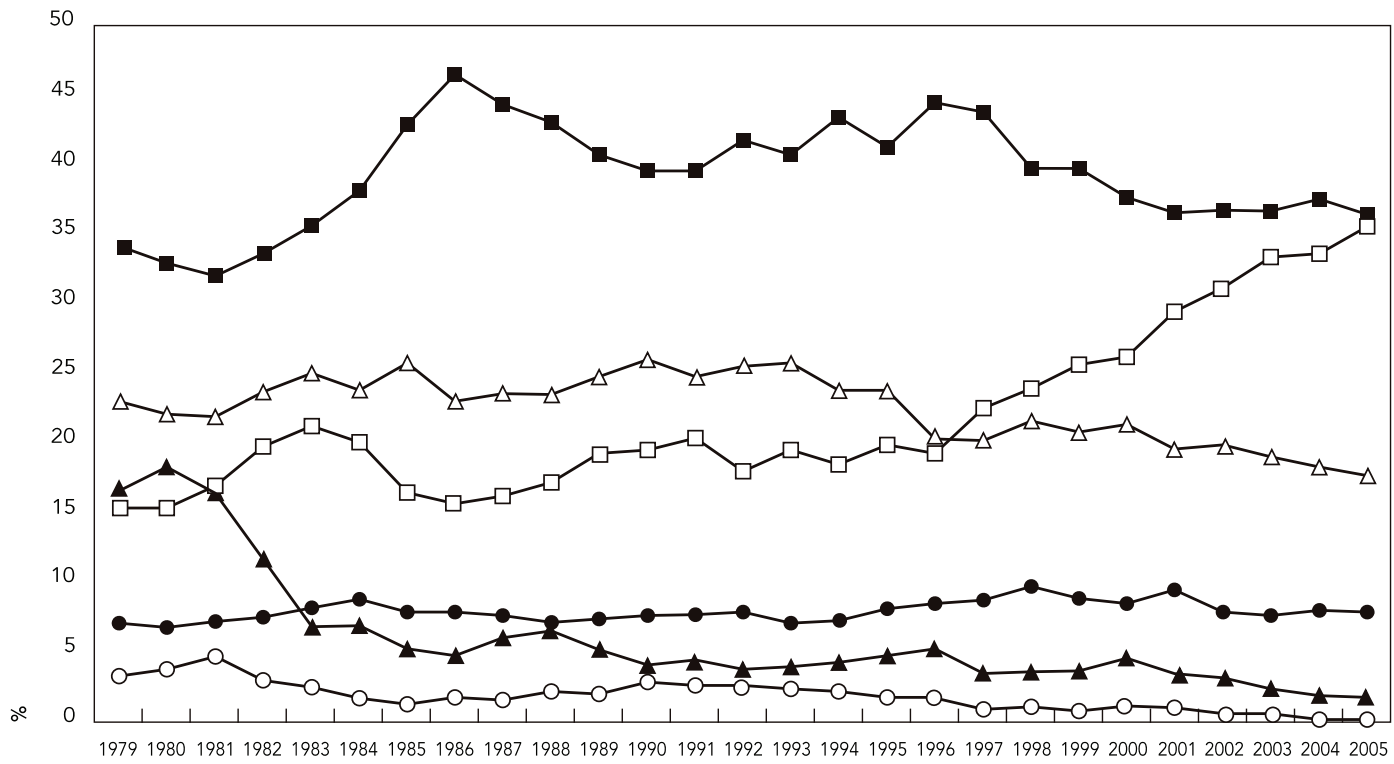

ção indeterminada por arma (de fogo ou branca) foi maior nos anos de 1981 (4,8\%) e 1990 (3\%), diminuindo constantemente até atingir 0,3\% em 2005 (Figura 2).

A melhora na qualidade dos registros das causas no SIM se confirma na diminuição dos coeficientes de mortalidade por eventos de intenção indeterminada de 14,9 óbitos por $100 \mathrm{mil}$ habitantes, em 1979/1981, para 2,0 em 2003/2005 (Tabela 1). A variação percentual anual foi de $-13,1 \%$ ao ano entre 1980 e $1985 ;-6 \%$ entre 1996 e 2000 e $-11 \%$ entre 2000 e 2004 (Tabela 1).

\section{Discussão}

Os resultados referentes às proporções de óbitos por eventos de intenção indeterminada indicam a boa qualidade dessas informações no Paraná. Em 1980, entre as causas externas, o percentual de óbitos por eventos de intenção indeterminada era de $22,3 \%$, diminuindo para $4,6 \%$ em 1999 , ao passo que para a Região Sul foram de $6,1 \%$ e 9,2\% nos dois anos, respectivamente 4 . Em 2005 o Paraná já tinha percentual de $2,1 \%$ enquanto o Estado de São Paulo apresentava $17 \%$ de óbitos por eventos de intenção indeterminada 5 entre as causas externas.
Outro achado que mostra a boa qualidade das informações de mortalidade por causas externas no Paraná é o comportamento inverso da tendência dos percentuais principalmente no início da década de 80, e a partir de 1996, quando foi observado aumento dos homicídios e queda na mortalidade por eventos de intenção indeterminada.

Cruz 6, comparando os coeficientes dos triênios 1979 a 1981 e 1990 a 1992, para o Rio de Janeiro, encontrou aumento de $90 \%$ nos coeficientes de mortalidade por homicídios com acréscimo dos óbitos de intenção indeterminada por lesão por arma de fogo e por arma branca. Diante disso e das evidências de que as mortes causadas por armas de fogo ou branca são, em sua grande maioria, homicídios de fato, o autor recomenda que, em estudos de mortalidade, os óbitos classificados como eventos de intenção indeterminada causados por armas sejam considerados homicídios. Para o Estado do Paraná, de acordo com os resultados deste estudo, tal procedimento não modificaria os níveis e a tendência ascendente dos homicídios após 1995, já que os coeficientes de mortalidade por eventos de intenção indeterminada são menores que um óbito por 100 mil habitantes. 
Coeficientes de mortalidade por causas externas (por 100 mil habitantes) e variação (\%) anual dos coeficientes por triênios. Paraná, Brasil, $1979-2005$.

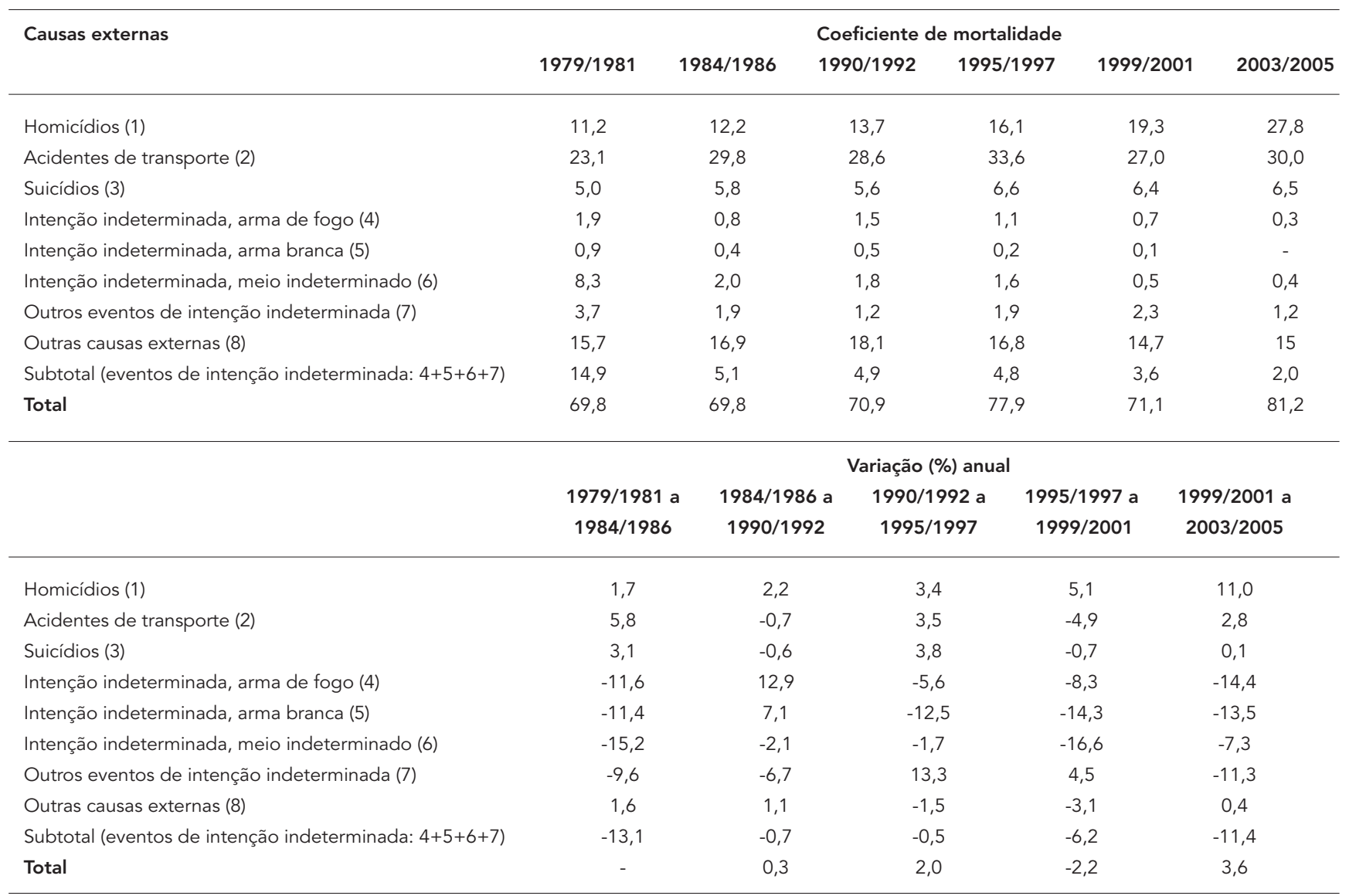

Com objetivo de melhorar as informações sobre mortalidade por causas externas, o Ministério da Saúde, desde 1996, passou a fazer constar na DO o campo VIII ("Prováveis circunstâncias da morte não natural”), informações de caráter estritamente epidemiológico. De responsabilidade do Instituto Médico Legal (IML) o preenchimento desse campo é baseado no boletim de ocorrência, informações de familiares, do hospital ou outra fonte. A variável 59 do campo VIII, em questão aberta, requer descrição do evento, incluindo o local de ocorrência. O preenchimento deste campo auxilia a codificação das causas de acordo com a classificação padronizada das circunstâncias geradoras de lesões e traumatismos mesmo que a descrição das causas na Parte I da DO esteja incompleta 7 .

No Paraná, o processamento do SIM passou a ser responsabilidade dos municípios em meados de 1993, facilitando o contato com os IMLs, médicos e imprensa, permitindo a corre- ção e classificação adequada da causa básica dos óbitos na DO. Por outro lado, a descentralização do SIM e conseqüente ampliação do número de codificadores de causas básicas, inicialmente, intensificaram a necessidade de realização de treinamentos, acompanhamento e supervisão do desempenho destes técnicos ${ }^{8}$. De fato, para o Paraná, foi observado ligeiro aumento na mortalidade proporcional e nos coeficientes de mortalidade por outros eventos de intenção indeterminada nos anos subseqüentes à municipalização do SIM, possivelmente por dificuldades iniciais das equipes em investigar e codificar as causas de óbito. A Secretaria de Estado da Saúde realizou então, em 2000, treinamento intensivo para os técnicos codificadores dos municípios e regionais de saúde. O impacto desse treinamento foi observado na queda considerável dos óbitos de intenção indeterminada, que reduziram de 5,9\% para apenas $2,1 \%$ dos óbitos por causas externas entre 2000 e 2005. 
Assim, é fundamental a realização de manutenção periódica das capacitações para técnicos, codificadores e equipe responsável pelo processamento dos dados, com novas orientações e aprimoramento dos conhecimentos, identificando e corrigindo as distorções nas DO assim como a digitação dos dados no sistema. Esta medida beneficiaria a vigilância de outros eventos importantes como a mortalidade infantil, materna e doenças de notificação compulsória, pois os codificadores e digitadores, na maioria dos casos, são responsáveis por todas as ações de vigilância da mortalidade no município e nas regionais de saúde do estado.

É necessário ainda empenho dos técnicos e gerentes do SIM junto aos IMLs para orientar os médicos legistas sobre a importância no preenchimento da DO considerando as circunstâncias que ocasionaram as lesões, e que se resolva a questão da legalidade da utilização das informa- ções da DO em processos judiciais, evitando que o médico legista possa ser penalizado indevidamente ${ }^{9}$.

A boa qualidade do banco de dados do SIM sobre causas externas no Estado do Paraná mostra que tem havido esforço e conscientização dos técnicos e codificadores municipais e estaduais de saúde no sentido de complementar e adequar as informações contidas nas DO para posterior inserção no sistema, gerando informação com potencial para auxiliar a elaboração de programas de prevenção e promoção da saúde. Além disso, este estudo traz à tona, mais uma vez, o problema do avanço dos homicídios nos últimos anos em nosso país e no Estado do Paraná, sendo necessários estudos mais detalhados para aprimorar o conhecimento sobre os acidentes e violências, permitindo melhor compreensão de seus determinantes e das possibilidades de intervenções.

\section{Resumo}

Foi analisada a qualidade do Sistema de Informações sobre Mortalidade (SIM) para óbitos por causas externas no Estado do Paraná, Brasil, entre 1979 e 2005, focalizando os eventos de intenção indeterminada. Os óbitos foram agrupados em acidentes de transporte, homicídios, suicídios, eventos de intenção indeterminada, e calculadas a mortalidade proporcional e a variação percentual anual dos coeficientes entre triênios. Os acidentes de transporte foram as causas mais freqüentes de óbito no período e, a partir de 1997, os homicídios passam à segunda posição. Os óbitos por eventos de intenção indeterminada por arma (de fogo ou branca) reduziram de 4,8\% em 1981 para 0,3\% em 2005. Os coeficientes dos eventos (todos) de intenção indeterminada passaram de 14,9 óbitos por 100 mil habitantes em 1979/1981 para 2,0 em 2003/2005. A variação percentual anual foi de $-13,1 \%$ entre $1980 e$ 1985; -6\% entre 1996 e 2000 e -11\% entre 2000 e 2004. Os resultados evidenciam a boa qualidade do SIM sobre causas externas no Estado do Paraná, permitindo análises com potencial para subsidiar ações de prevenção e promoção da saúde.

Causas Externas; Homicídio; Mortalidade; Sistemas de Informação

\section{Colaboradores}

E. M. K. Lozada participou na concepção, análise dos dados e elaboração do manuscrito. T. A. F. Mathias, T. Aidar e S. M. Andrade colaboraram na análise e interpretação dos dados elaboração e revisão do manuscrito.

\section{Agradecimentos}

Ao Departamento de Ciência e Tecnologia (DECIT), Secretaria de Ciência, Tecnologia e Insumos Estratégicos (SCTIE) do Ministério da Saúde e ao Conselho Nacional de Desenvolvimento Científico e Tecnológico (CNPq), pelo apoio financeiro (CT-Saúde/CNPq 24/2004 - processo $n^{\circ}$. 505.875/2004-7). 


\section{Referências}

1. Minayo MCS, Souza ER, organizadores. Violência sob o olhar da saúde: a infrapolítica da contemporaneidade brasileira. Rio de Janeiro: Editora Fiocruz; 2003.

2. Minayo MCS. The inclusion of violence in the health agenda: historical trajectory. Ciênc Saúde Coletiva 2006; 11:375-83.

3. Aidar T. O impacto das causas violentas no perfil de mortalidade da população residente no município de Campinas: 1980 a 2000. Rev Bras Estud Popul 2003; 20:281-302.

4. Mello Jorge MHP, Gotlieb SLD, Laurenti R. A saúde no Brasil: análise do período 1996 a 1999. Brasília: Organização Pan-Americana da Saúde; 2001.

5. Gawryszewski VP, Morita M, Hidalgo NT, Valencich DMO, Brumini R. A mortalidade por causas externas no Estado de São Paulo em 2005. Boletim Epidemiológico Paulista 2006; 3:2-7.

6. Cruz OG. Homicídios no Estado do Rio de Janeiro: análise da distribuição espacial e sua evolução [Dissertação de Mestrado]. São Paulo: Faculdade de Saúde Pública, Universidade de São Paulo; 1996.
7. Mello Jorge MHP, Cascão AM, Silva RC. Acidentes e violências: um guia para o aprimoramento da qualidade de sua informação. São Paulo: Centro Brasileiro de Classificação de Doenças em Português; 2003. (Série Divulgação, 10).

8. Mello Jorge MHP, Laurenti R, Gotlieb SLD. Análise da qualidade das estatísticas vitais brasileiras: a experiência de implantação do SIM e do SINASC. Ciênc Saúde Coletiva 2007; 12:643-54.

9. Matos SG, Proietti FA, Barata RCB. Confiabilidade da informação sobre mortalidade por violência em Belo Horizonte, MG. Rev Saúde Pública 2007; 41:76-84.

Recebido em 29/Abr/2008

Versão final reapresentada em 28/Ago/2008

Aprovado em 20/Out/2008 